\title{
Organisms Isolated from Urine in a Mixed Paediatric Population
}

Rosalind Saunders', Simon Hewson ${ }^{1}$, Srini Bandi²

${ }^{1}$ Department of Clinical Microbiology, ${ }^{2}$ Leicester Children's Hospital; University Hospitals of Leicester NHS Trust, Leicester, UK

\section{Background}

The Leicester Microbiology Laboratory serves a population of approximately 200,000 children, and receives urine samples from Leicester Children's Hospital and community general practices.

UK-wide surveillance data for antimicrobial resistance in samples from children is not routinely published. The aim of this retrospective survey was therefore to focus on paediatric urine cultures in order to inform local empirical paediatric antimicrobial prescribing.

\section{Methods}

The microbiology laboratory results database was searched for all urine samples taken from paediatric patients (age <16) over a 1-year period (1 March 2016 - 28 February 2017). Any discarded samples (e.g. leaking, unlabelled) were excluded.

Data extracted included demographic data, requesting location (hospital/community) and microbiology result including organism name and antimicrobial susceptibilities.

Sensitivity testing in our laboratory for Gram negative organisms consists of a first-line panel consisting of five clinicallyused antibiotics plus Cefpodoxime (used as a marker of resistance). Samples are tested against a further six antibiotics if the initial test is resistant to Cefpodoxime, or on medical request (e.g. multiple allergies).

\section{Results}

Data for 5928 paediatric urine samples were analysed from 4458 unique patients. $1826(31 \%)$ were taken within the acute hospital setting, with 4102 (69\%) being sent from community sources.

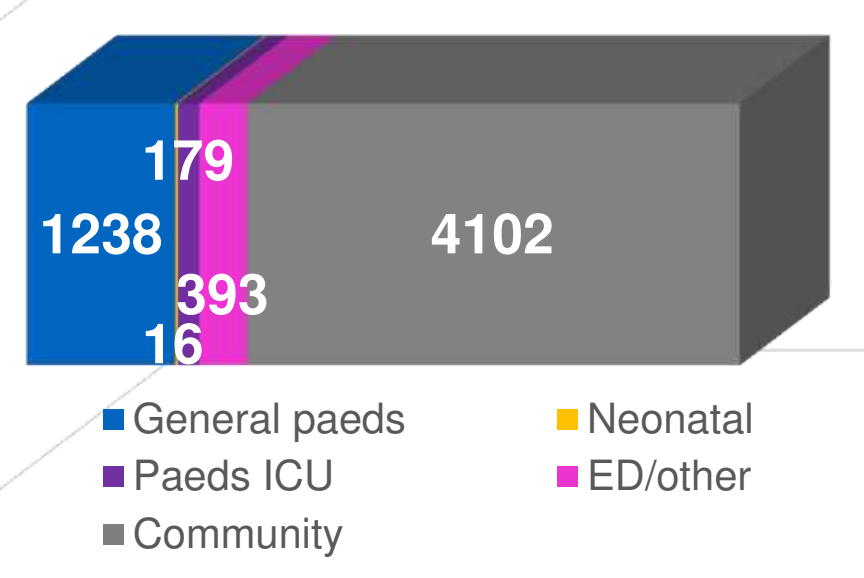

Overall, 2530 (43\%) urine samples were culture-positive, although 404 samples had mixed bacterial flora ( $\geq 3$ organisms) consistent with faecal contamination. Samples with more than two organisms do not undergo further identification or sensitivity testing in the laboratory.

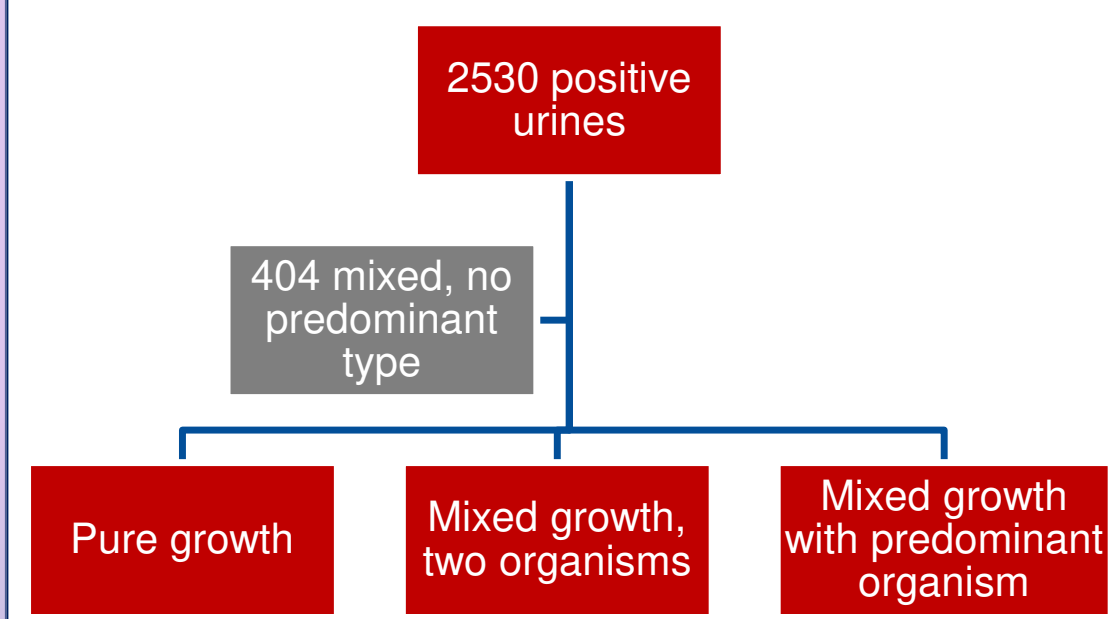

Therefore 2126 samples (36\% of total) had susceptibility testing carried out. Because some samples had mixed growth, we obtained identification and sensitivity results from 2733 organisms in total.

\section{Organisms \& Sensitivities}

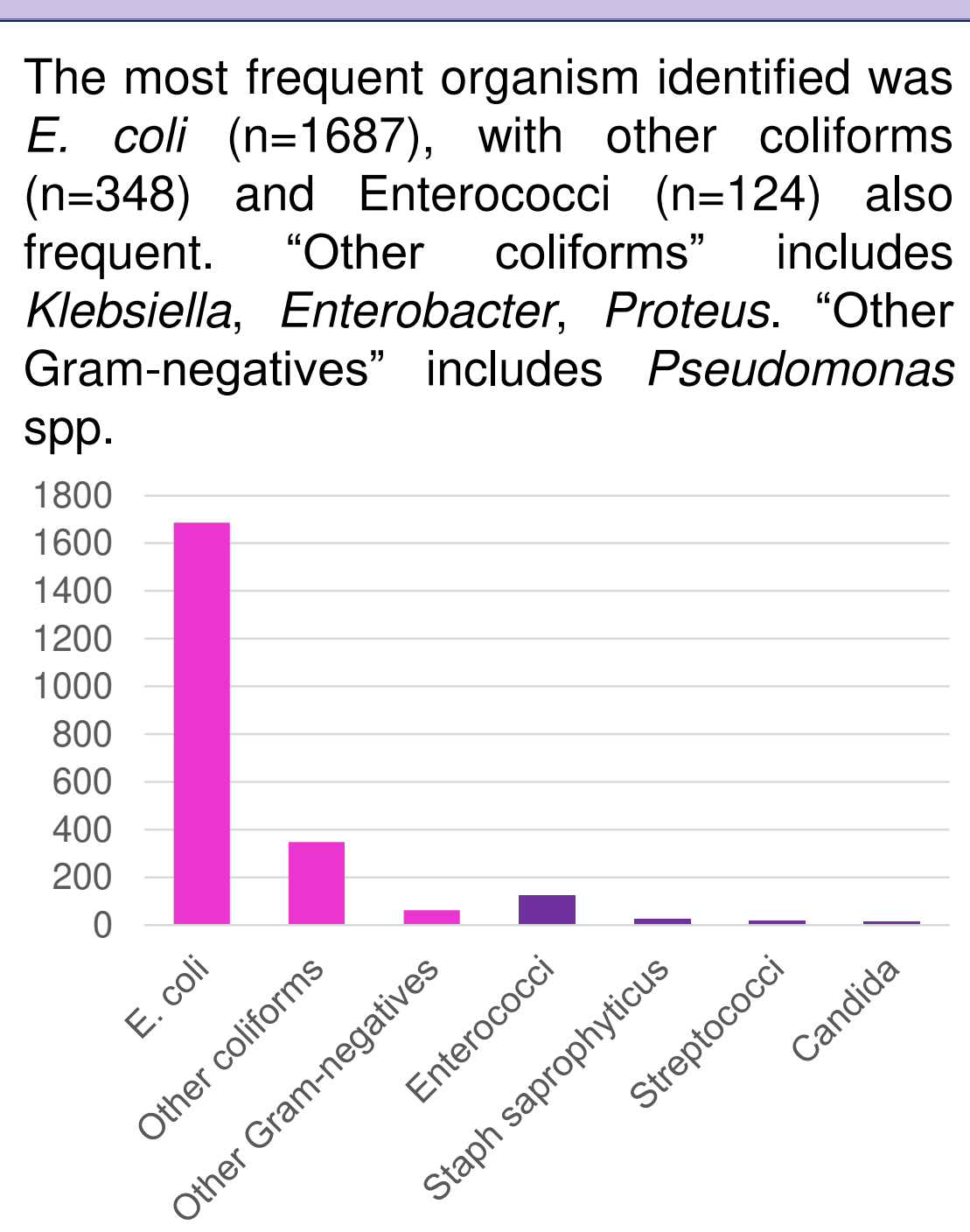

Sensitivity testing for $E$. coli First line panel: $n=1687$

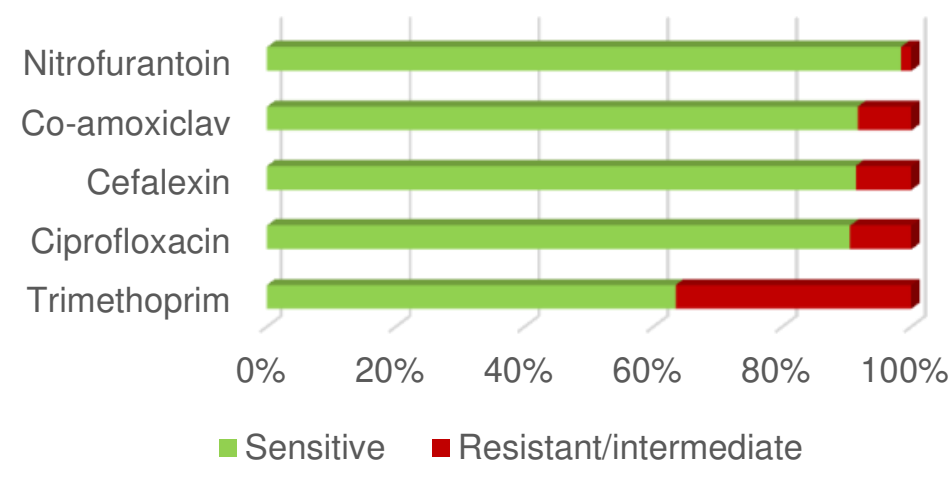

Second line panel: $n=122$

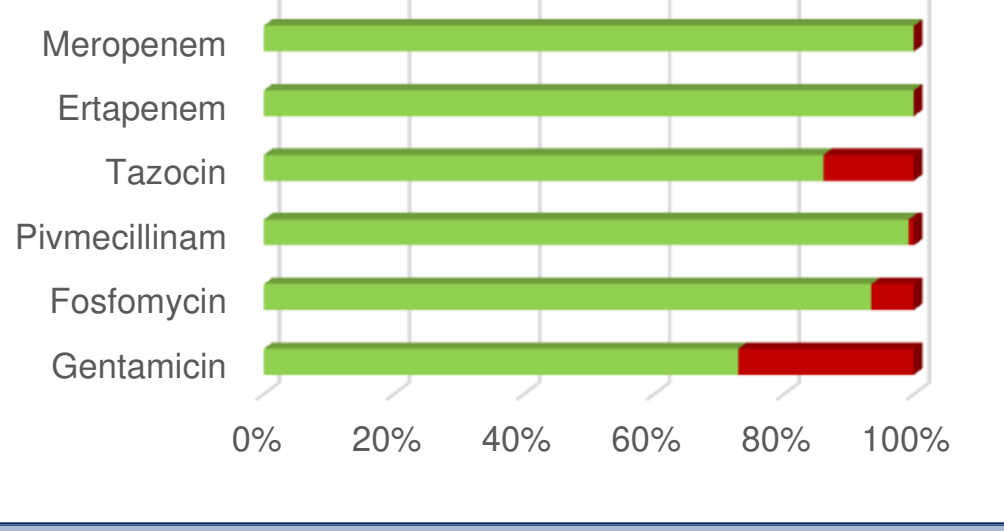

University Hospitals of Leicester WHS

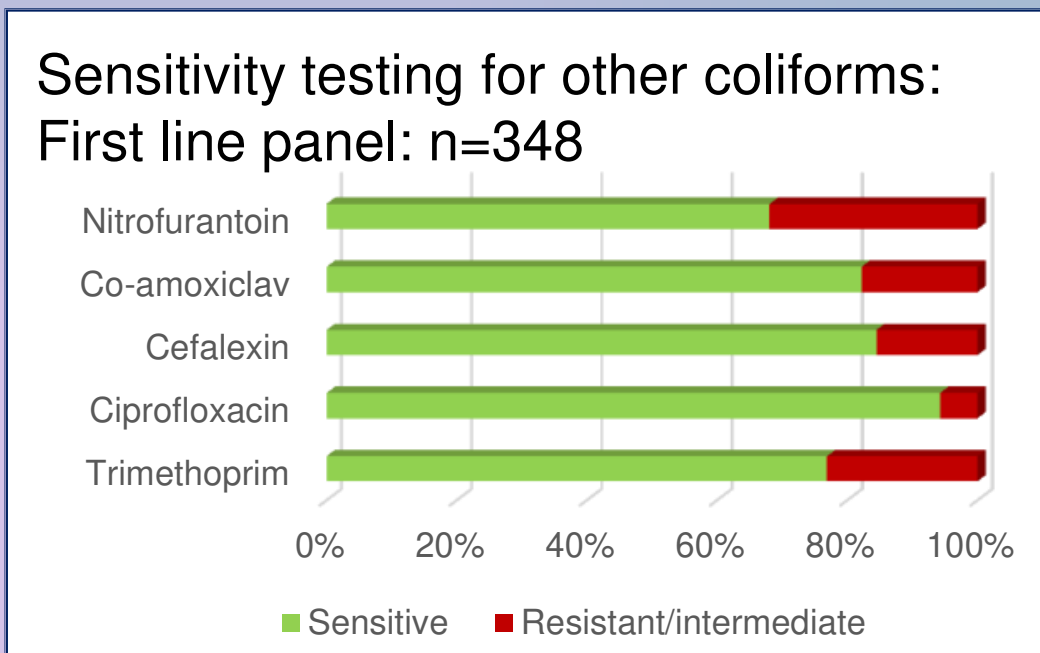

Sensitivity testing for Enterococci:

First line panel: $n=124$

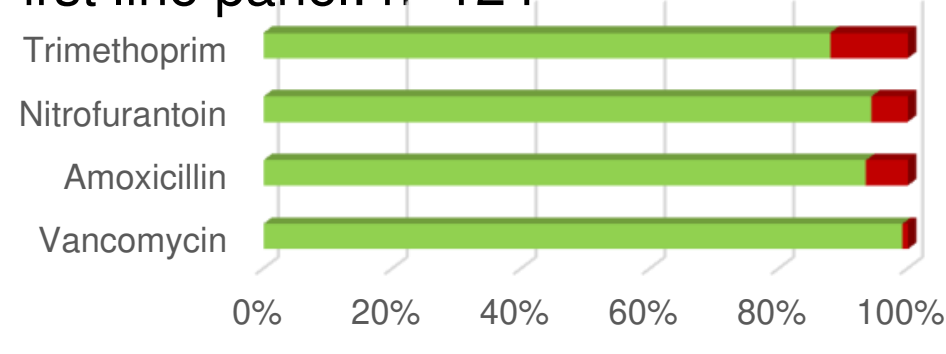

\section{Conclusions}

Resistance rates of $E$. coli to trimethoprim in this study were $37 \%$, suggesting that our use of trimethoprim as first line empirical therapy for urinary tract infection in children needs review. Multi-resistant organisms in our local paediatric population are not yet problematic, and several oral alternatives are available.

However, decision-making is complicated by a number of factors. Sampling bias may be present - despite current guidance to send a sample for culture before treatment begins, an unquantified number of samples in this study may represent samples taken in response to treatment failure. Moreover, empirical therapy also needs to take into account other organisms, as well as side effect profile, palatability/compliance and cost. 